\title{
Effect of Graphene and Carbon Nanotubes on the Thermal Conductivity of WC-Co Cemented Carbide
}

\author{
Kui Chen ${ }^{\mathrm{a}}$, Wenkai Xiao ${ }^{\mathrm{b}^{*}}$, Zhengwu $\mathrm{Li}^{\mathrm{b}}$, Jiasheng $\mathrm{Wu}^{\mathrm{b}}$, Kairong Hong ${ }^{\mathrm{a}}$, Xuefeng Ruan ${ }^{\mathrm{c}}$
}

a. State Key Laboratory of Shield Machine and Boring Technology;

b.School of Power and Mechanical Engineering, Wuhan University;

c. Research Center of Nanoscience and Technology, Wuhan University

\begin{abstract}
In recent years, it has been found in engineering practice that the service life of cemented carbide shield machine tools used in uneven soft and hard strata is substantially reduced. The study found that thermal stress is the main reason for the failure of cemented carbide shield tunneling tools when shield tunneling is carried out in uneven soft and hard soil. To maintain the hardness of cemented carbide, improving the thermal conductivity of the shield machine tool is of great importance for prolonging its service life and reducing engineering costs. In this paper, graphene and carbon nanotubes were mixed with WC-Co powder and sintered by SPS (Spark Plasma Sintering). The morphology was observed by using scanning electron microscopy (SEM) and energy dispersive X-ray spectroscopy (EDS). A Rockwell hardness tester and bending strength tester were used to test hardness, bending strength and thermal conductivity. The results show that adding trace graphene or carbon nanotubes can increase the bending strength of the cemented carbide by approximately $50 \%$ while keeping the hardness of the cemented carbide unchanged. The thermal conductivity of the cemented carbide can be increased by $10 \%$ with the addition of $0.12 \%$ graphene alone.
\end{abstract}

Keywords: Cemented carbide; Carbon nanotubes; Graphene; Thermal conductivity; Bending strength 


\section{Introduction}

Cemented carbide has sufficient hardness, wear resistance, and toughness to avoid brittle fracture. It has been applied very successfully in shield cutting tools. However, when shield tunneling in uneven soft and hard underlying soil, thermal stress and other factors substantially reduce the service life of cutting tools. (The average working temperature of the cutter in the shield machine is $500^{\circ} \mathrm{C}$, sometimes $1500^{\circ} \mathrm{C}$ in extreme circumstances $)^{[1][2]}$

Current research shows that the main failure modes of cemented carbide cutters in shield machines are fracture and wear. The reason for failure is that under the simultaneous action of mechanical stress and thermal stress, microcracks are formed and then propagate until the failure of the cemented carbide tools occurs. ${ }^{[3]}$. Braiden et al. suggested that thermal stress leads to tool cracking, which leads to tool propagation and fracture, resulting in a sharp decline in the service life of cemented carbide under thermal stress. It was also proved by experiments that tool life was significantly affected by the region of thermal strain and the number of thermal cycles during intermittent cutting. Error! Reference source not found. Beste, Coronel and Jacobson et al. prepared slices of the bit surface for analysis using focused ion beam and electron beam thinning. The samples of the bit surface after drilling were analyzed with a TEM (transmission electron microscope). ${ }^{\text {Error! }}$ Reference source not found. The Co bonding phase was confirmed to melt first at high temperature, while WC did not. The results showed that the weakness of cemented carbide tools may be mainly due to the partial melting or softening of the bonding phase due to an insufficient melting point and insufficient thermal conductivity of the bonding phase at high temperature; the cemented carbide then fails due to thermal stress. ${ }^{[5]}$

Therefore, if a good method can be found to improve the thermal conductivity while maintaining the hardness of the cemented carbide, the temperature of the tool will be greatly reduced, and the service life of the tool will be increased. Graphene and carbon nanotubes have attracted much attention in recent years due to their very high thermal conductivity and strength. We attempted to use the excellent properties of graphene and carbon nanotubes to improve the high-temperature properties of cemented carbides. If graphene and carbon nanotubes can be evenly dispersed in the Co phase of cemented carbide, then the dispersed graphene and carbon nanotubes in the cemented carbide can form effective high thermal conductivity channels, improving their thermal conductivity. Moreover, the second phase dispersion strengthening principle can be used to improve the fracture resistance of the cemented carbide matrix to ensure that the cemented carbide retains a high hardness while improving the thermal conductivity. In the field of ceramics and metals, Faming Zhang, Zhiqiang Huang and others added nanophases (carbon nanotubes and nano- $\mathrm{Al}_{2} \mathrm{O}_{3}$ ) to cemented carbides, which played a role in strengthening and toughening the Co phase ${ }^{[7][8]}$.

However, there are no studies on the effects of graphene and carbon nanotubes on the thermal conductivity of WC-Co cemented carbide. In this paper, WC-Co cemented carbide with different 
graphene and carbon nanotube contents was prepared by a powder metallurgy process. The effects of the graphene and carbon nanotubes on the thermal conductivity, hardness and strength were investigated.

\section{Experimental}

\subsection{Materials}

The primary tungsten carbide powder used in this study had a particle size of $5 \mu \mathrm{m}$ and a purity of $99.9 \%$. The nano-cobalt powder had a particle size of $50 \mathrm{~nm}$, a purity of $99.9 \%$, a surface area of 40-60 m3/g, and a density of $8.9 \mathrm{~g} / \mathrm{cm}^{3}$. The single-walled carbon nanotubes (SWCNTs) had a thickness of $0.8 \mathrm{~nm}$, diameter of $0.5-2 \mu \mathrm{m}$, purity of $99 \%$, single-layer rate of $80 \%$, and thermal conductivity of $5800 \mathrm{~W} / \mathrm{mK}$. The specific surface area of monolayer graphene is greater than 380 $\mathrm{m}^{2} / \mathrm{g}$, the purity is greater than $90 \%$, and the thermal conductivity is $4000 \mathrm{~W} / \mathrm{mK}$. In this study, graphene and carbon nanotubes were added as a percentage of Co content, and the content of Co in the cemented carbide samples was $6 \mathrm{wt} \%$. The composition of the test samples are shown in Table 1 (mean values of three samples in each group).

Table 1 The composition of the test samples

\begin{tabular}{ccccc}
\hline Sample group number & 0 & 1 & 2 & 3 \\
\hline Graphene & $0 \%$ & $0.06 \%$ & $0 \%$ & $0.12 \%$ \\
\hline Carbon nanotubes & $0 \%$ & $0.06 \%$ & $0.12 \%$ & $0 \%$ \\
\hline
\end{tabular}

A planetary ball mill was used to mix the powder. According to the force analysis and calculation of the ball in the planetary ball mill, it was concluded that with $\mathrm{r}=0.0325 \mathrm{~m}$, the rotational speed below $166 \mathrm{r} / \mathrm{min}$ would not centrifuge. The ball milling speed was $110 \mathrm{r} / \mathrm{min}$, and the milling time was 4 hours. Ethanol was used as the medium. ${ }^{[9]}$ After powder mixing and dispersing, the mixed powder was vacuum dried in a vacuum drying furnace. The dried powder was poured into high strength graphite jig. A sintered cylindrical sample with a diameter of $20 \mathrm{~mm}$ was prepared by SPS sintering equipment. The circular sintered samples were cut into one square and two strips by a wire cutting machine. The size of the block was 10x10x3 mm, and the lengths were $19 \times 2 \times 3 \mathrm{~mm}$ and $17 \times 2 \times 3 \mathrm{~mm}$. The diamond sample was used to measure the thermal diffusivity and hardness, and the strip sample was used to measure the bending strength and then for morphology analysis. Because of the high hardness of the sample, the upper and lower surfaces of the sample were burnished with diamond abrasive discs.

\subsection{Testing of WC-Co Cemented Carbide Samples}

Because of the small size of the sintered sample in this experiment, the thermal conductivity of the material cannot be measured directly but can be calculated by measuring the thermal diffusivity and other parameters. Therefore, a TC-7000H laser thermal constant tester was used to measure the thermal diffusivity of WC-Co cemented carbide samples by a laser method. The 
specific heat capacity of WC-Co cemented carbide samples was measured by differential scanning calorimetry. Sample quality was measured with an electronic scale, and sample volume was measured with the Archimedes method. The bending strength of the sample was measured by a three-point test using a bending strength tester. The hardness of the sample was measured by a Rockwell hardness tester. The morphology of the samples was observed by MIRA 3 LMH field emission scanning electron microscope, and EDS (energy dispersive spectroscopy) was carried out with an Aztec Energy Standard X-MaxN 20 instrument.

Table 2 Thermal Diffusion Coefficient of WC-Co Cemented Carbide

\begin{tabular}{ccccc}
\hline Sample group number & 0 & 1 & 2 & 3 \\
\hline Thermal diffusivity $\left(\mathrm{mm}^{2} / \mathrm{s}\right)$ & 16.736 & 16.221 & 17.555 & 18.021 \\
\hline
\end{tabular}

\section{Results and discussion}

\subsection{Thermal Conductivity of WC-Co Cemented Carbide}

After testing the thermal diffusivity, we obtained the following data at $500^{\circ} \mathrm{C}$. The volume and mass were determined for each sample, and the average density of the sample group was calculated.

Table 3 Density of WC-Co Cemented Carbide

\begin{tabular}{ccccc}
\hline Sample group number & 0 & 1 & 2 & 3 \\
\hline Mass $(\mathrm{g})$ & 1.9003 & 2.9412 & 2.8941 & 2.6838 \\
\hline Volume $\left(\mathrm{cm}^{3}\right)$ & 0.13 & 0.19 & 0.20 & 0.18 \\
\hline Density $\left(\mathrm{g} / \mathrm{cm}^{3}\right)$ & 14.62 & 15.48 & 14.47 & 14.91 \\
\hline
\end{tabular}

The difference in specific heat capacity between the three groups of samples was very small. Because of equipment limitations, only the specific heat capacity data between room temperature and $400^{\circ} \mathrm{C}$ could be measured. Therefore, the specific heat capacity data at $500^{\circ} \mathrm{C}$ could be estimated from the linear relationship between the specific heat capacity and temperature change. The specific heat capacities of the three groups of samples were estimated to be approximately $0.23 \mathrm{~J} /\left(\mathrm{g} \cdot{ }^{\circ} \mathrm{C}\right)$ at $500^{\circ} \mathrm{C}$ by linear fitting.
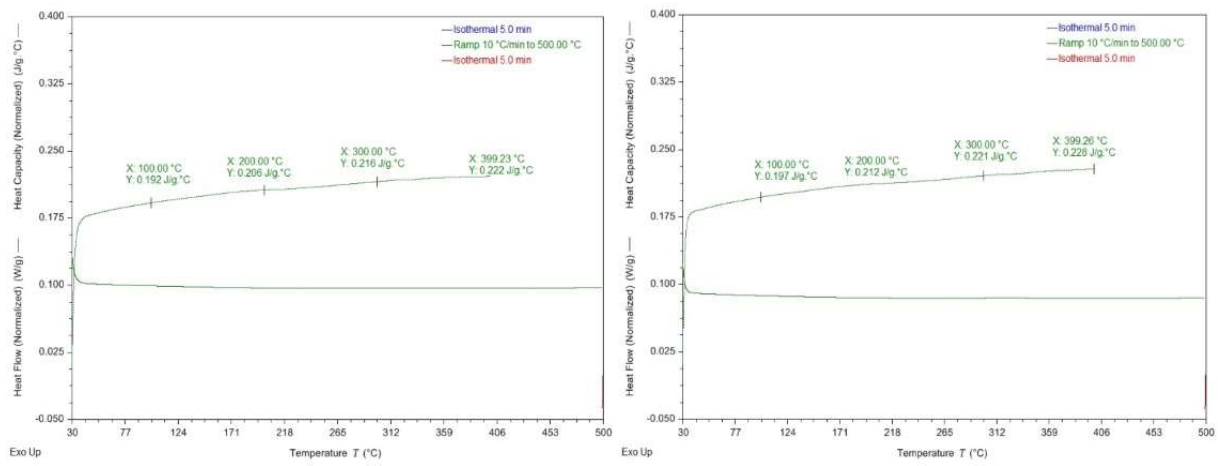

Fig. 1 Specific Heat Capacity Data Between Room Temperature and $400^{\circ} \mathrm{C}$ 
Thermal conductivity can be calculated by the following formula:

$$
\lambda=\mathrm{a} * \mathrm{Cp} * \mathrm{P}(\mathrm{Cp}=0.23)
$$

Table 4 Thermal Conductivity of WC-Co Cemented Carbide

\begin{tabular}{ccccc}
\hline Sample group number & 0 & 1 & 2 & 3 \\
\hline Thermal diffusivity & 16.736 & 16.221 & 17.555 & 18.021 \\
\hline Density $\left(\mathrm{g} / \mathrm{cm}^{3}\right)$ & 14.62 & 15.48 & 14.47 & 14.91 \\
\hline $\begin{array}{c}\text { Thermal Conductivity } \\
\mathrm{W} /(\mathrm{m} \cdot \mathrm{K})\end{array}$ & 56.28 & 57.76 & 58.41 & 61.83 \\
\hline
\end{tabular}

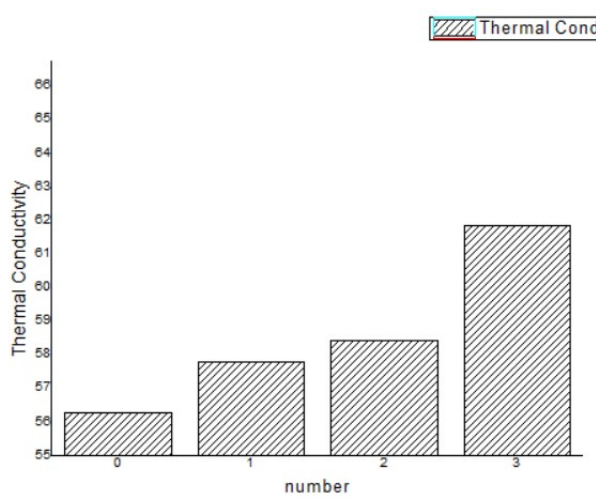

Fig. 2 Thermal Conductivity of WC-Co Cemented Carbide

Fig. 2 shows that the thermal conductivity of the samples increased to varying degrees after adding graphene and carbon nanotubes. The thermal conductivity of the cemented carbide samples with $0.06 \%$ carbon nanotubes and $0.06 \%$ graphene increased by $2.63 \%$ compared with that of the original samples. The thermal conductivity of the cemented carbide samples with $0.12 \%$ carbon nanotubes was $3.78 \%$ higher than that of the original samples, and the thermal conductivity of the cemented carbide samples with $0.12 \%$ graphene was $9.86 \%$ higher than that of the original samples. It is noteworthy that the thermal conductivity of the $0.12 \%$ graphene samples increased substantially, with a nearly $10 \%$ increase. This shows that graphene has the best effect on the thermal conductivity of cemented carbide compared to that of carbon nanotubes. This may be due to the following factors. First, the specific surface area of graphene and carbon nanotubes is large, although the mass fraction is only $0.12 \%$, and the actual volume fraction may be higher. Second, some types of graphene distributions can bridge the interface between WC and Co and reduce the thermal resistance. According to the theory of contact thermal resistance, the thermal resistance between the solid interfaces will be produced due to incomplete contact. Jin peng's research proved that in WC-Co cemented carbide, the more WC grain boundaries there were, the worse the thermal conductivity, so the interface is one of the main reasons for the larger thermal resistance ${ }^{[11]}$. The addition of fillers with good thermal conductivity between the interfaces could reduce the contact thermal resistance ${ }^{[10]}$. Therefore, the addition of graphene reduced the thermal resistance between the WC and Co interfaces. Third, as a crystalline two-dimensional single-layer of carbon, 
the two-dimensional structure of graphene can significantly reduce the boundary scattering of phonons at the grain boundary ${ }^{[12]}$. WC-Co cemented carbide conducts heat through the movement of Co free electrons and the vibration of the WC lattice structure. The phonon boundary scattering at WC grain boundaries is substantial, and the two-dimensional structure of graphene can significantly reduce the phonon boundary scattering at the grain boundaries. Fourth, according to the law of minimum thermal resistance, graphene with a certain filling rate can form an effective high thermal conductivity network, increase high thermal conductivity channels, and greatly enhance the thermal conductivity of materials. In summary, graphene is very effective in improving the thermal conductivity of WC-Co cemented carbide, and therefore a minute amount of dispersed graphene can greatly improve the thermal conductivity.

\subsection{Hardness Testing of WC-Co Cemented Carbide}

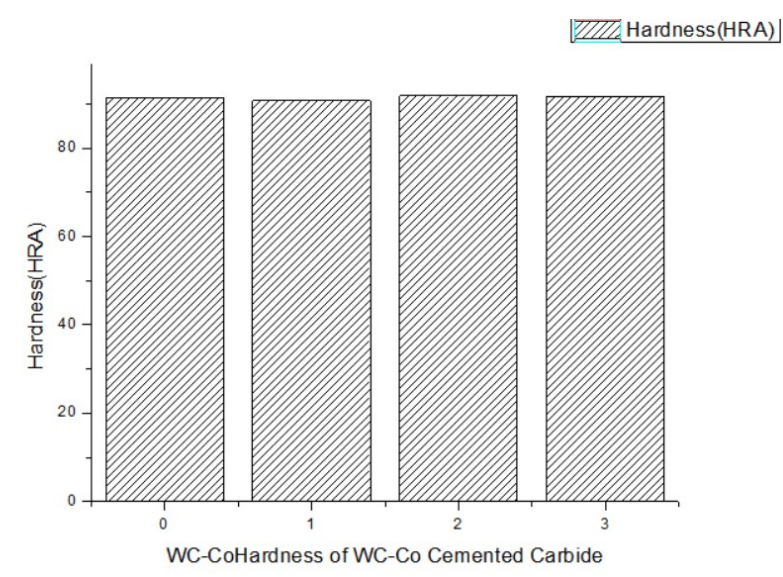

Fig. 3 Hardness of WC-Co Cemented Carbide

It can be seen from the diagram that the hardness of the sample remains basically unchanged.

\subsection{Bending Strength Test of WC-Co Cemented Carbide}

Using the bending strength tester, the bending strength of the sample was measured by a three-point test method. The data are as follows.

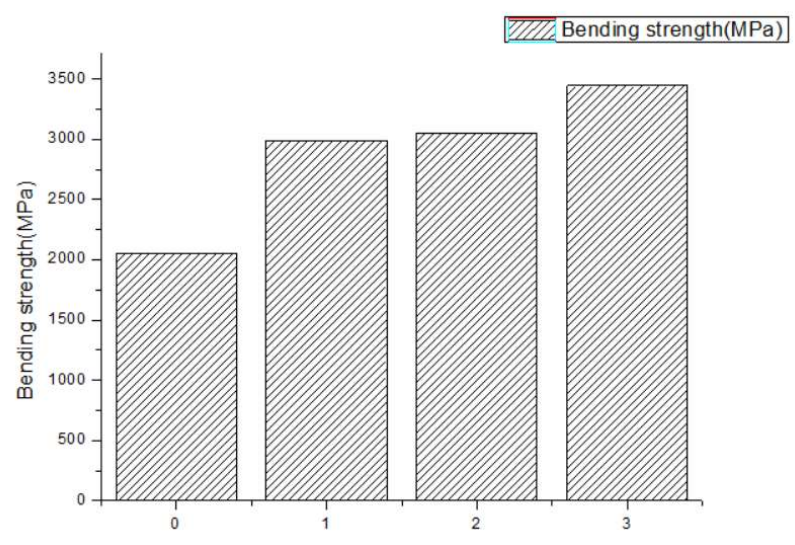

Fig. 4 Bending Strength of WC-Co Cemented Carbide

According to Fig. 4, the bending strength of the samples with $0.06 \%$ graphene $+0.06 \%$ carbon nanotubes increased by $48.73 \%$, the bending strength of the samples with $0.12 \%$ CNTs increased 
by $45.76 \%$, and the bending strength of the samples with $0.12 \%$ graphene increased by $67.84 \%$. After graphene was dispersed in the cemented carbide bonding phase, its flexural strength was substantially improved. Because graphene has a small size and high hardness, it plays a role in the second phase dispersion strengthening in the weak Co phase.

Cracks in cemented carbides usually propagate in the weak adhesive cobalt phase and ultimately lead to fracture failure. When a crack propagates in a cobalt phase with insufficient yield strength and encounters hard graphene particles or carbon nanotubes, the crack is forced to bypass them because it cannot pass through. As a result, the crack path becomes longer. Therefore, crack propagation and fracture are more difficult in cemented carbide materials because graphene and carbon nanotubes absorb more external energy.

\subsection{SEM Analysis of WC-Co Cemented Carbide}

The secondary electron and backscattered electron images of WC-Co cemented carbide with $0.12 \%$ graphene were obtained in this experiment. The location of Co was found by comparing the difference between the secondary electron image and backscattered electron image. EDS was also carried out.

\subsubsection{Backscattered and Secondary Electron Images of WC-Co Cemented Carbide Samples}

The yield of backscattered electrons is positively correlated with the atomic number. Thus, the bright part in a secondary electron image and the dark part in a backscattered electron image for the same field of view contain components with a lower mass contrast, which in this case, can be judged to be Co.

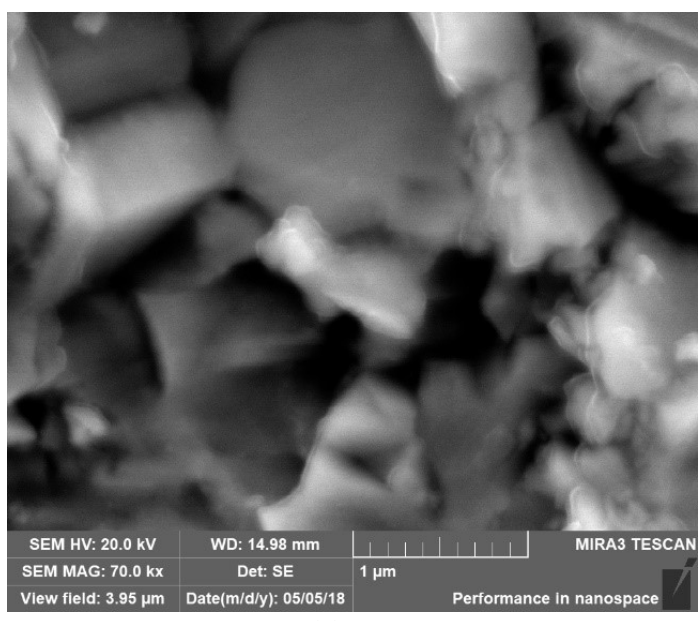

(a)

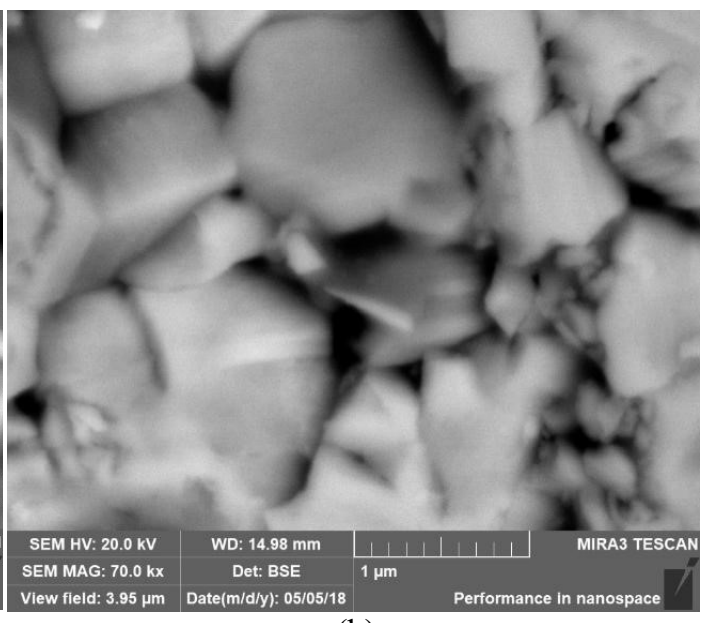

(b)

Fig. 5 (a) Secondary electron image (b) Backscattered electron image

\subsubsection{Energy Spectrum Analysis of WC-Co Cemented Carbide Samples}

Graphene and carbon nanotubes were basically distributed in the Co phase; thus, EDS was used to analyze the position of Co to find the location of the graphene and carbon nanotubes. 


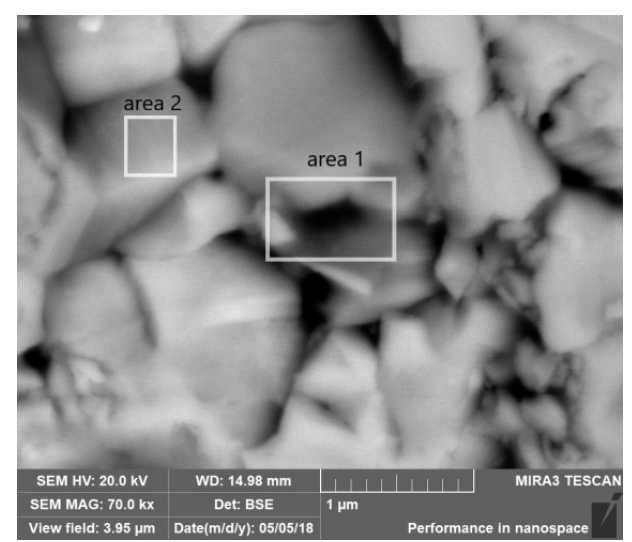

(a)

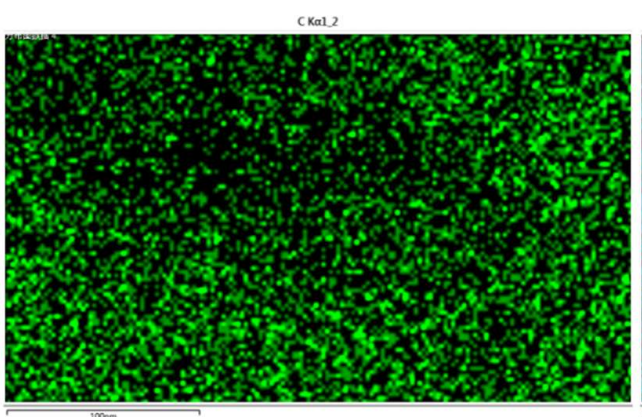

(c)

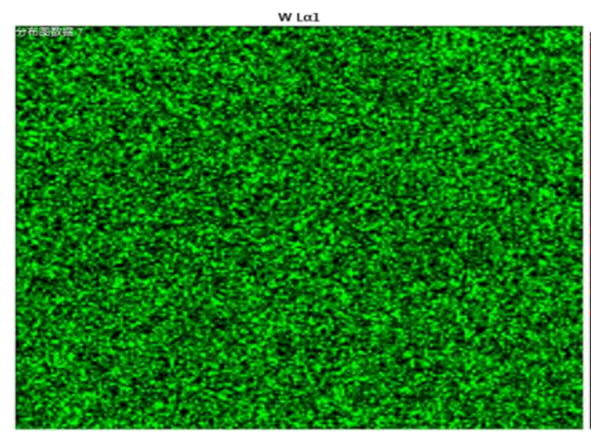

(e)

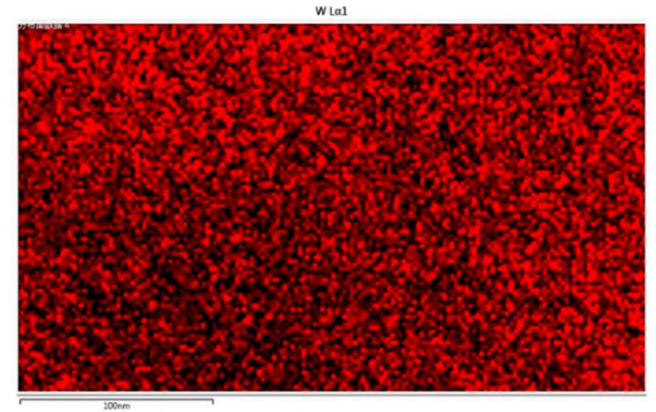

(b)

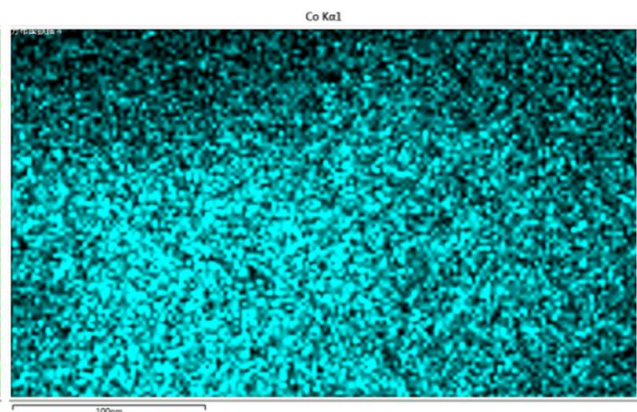

(d)

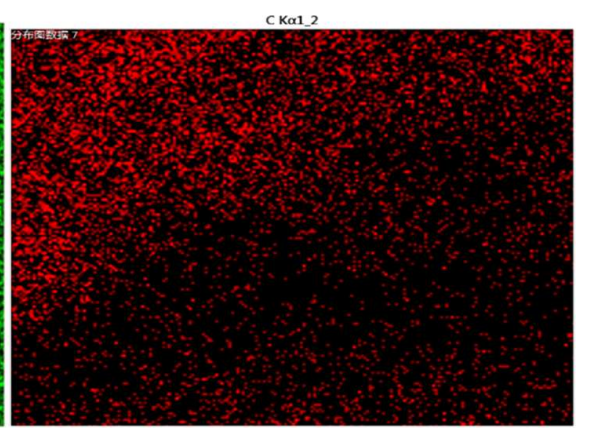

(f)

Fig. 6 (a) Image of area used for EDS analysis. (b) Distribution of W content in Area 1. (c) Distribution of Co content in Area 1. (d) Distribution of C content in Area 1. (e) Distribution of W content in Area 2. (f) Distribution of $\mathrm{C}$ content in Area 2.

The analysis of the composition distribution in the area shown in Fig. 6 (a) indicated that the W component in the lower left corner of the region was relatively small, so the $\mathrm{C}$ content in the WC was relatively small. The Co content was concentrated, indicating that the region in the lower left corner was a block of Co. The region in the lower left corner was relatively high in carbon content, indicating that graphene was more distributed in Co. 


\subsection{Finite Element Simulation of Heat Transfer Process of Gear Cutter of Shield Machine}

$$
\frac{\partial t}{\partial \tau}=\frac{\lambda}{\rho_{C}}\left(\frac{\partial^{2} t}{\partial x^{2}}+\frac{\partial^{z} t}{\partial y^{2}}+\frac{\partial^{z} t}{\partial z^{2}}\right)
$$

The time integration for transient solid heat transfer in ABAQUS is based on the backward difference method.

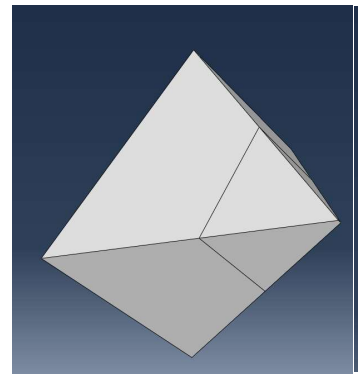

(a)

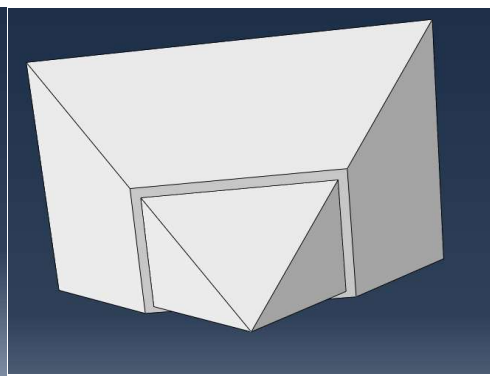

(b)

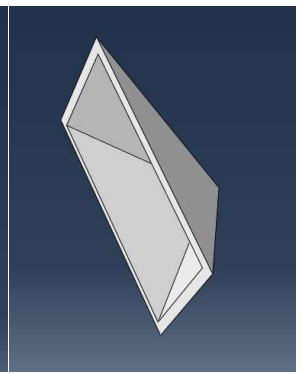

(c)

Fig. 7 Model of the Tooth Cutter Element for a Shield Machine

Fig. 7 (a) is a model of a tooth cutter element for a shield machine with a side length of $30 \mathrm{~mm}$. It is divided into two parts. Fig. 7 (c) is a shell with a thickness of $0.5 \mathrm{~mm}$. The remaining part is treated as a whole, as shown in Fig. 7 (b). Next, set the temperature of the part in Fig. 7 (c) to 1500 degrees and the part in Fig. 7 (b) to 500 degrees. It is assumed that the surface of the gear cutter is adiabatic and does not exchange heat with the outside world. The mesh of the gear cutter was divided by using the DC3D4 element type, and the cell number is $1.3 \mathrm{e}^{5}$. By inputting the density, specific heat capacity and thermal conductivity of the original sample and the sample with $0.12 \%$ graphene into the calculation, the dynamic heat transfer process of the teeth can be obtained.

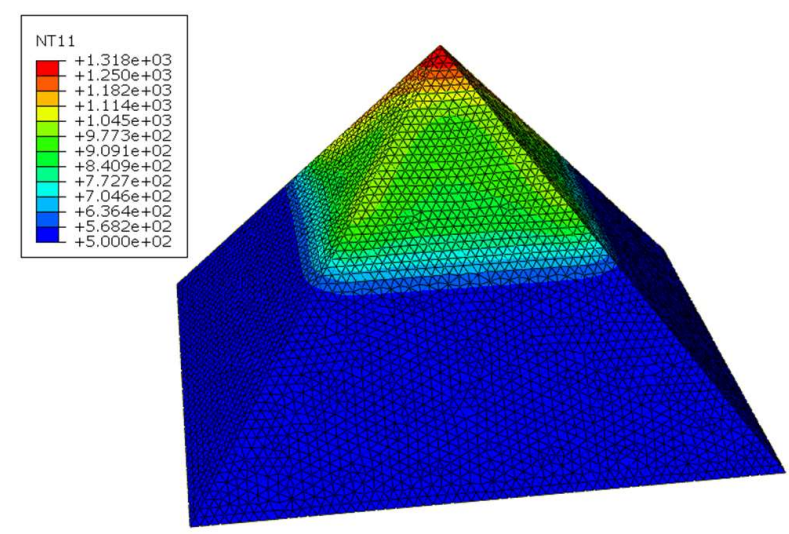

Fig. 8 Fig. 8 Temperature nephogram of samples with graphene after $1 \mathrm{~s}$ heat transfer 


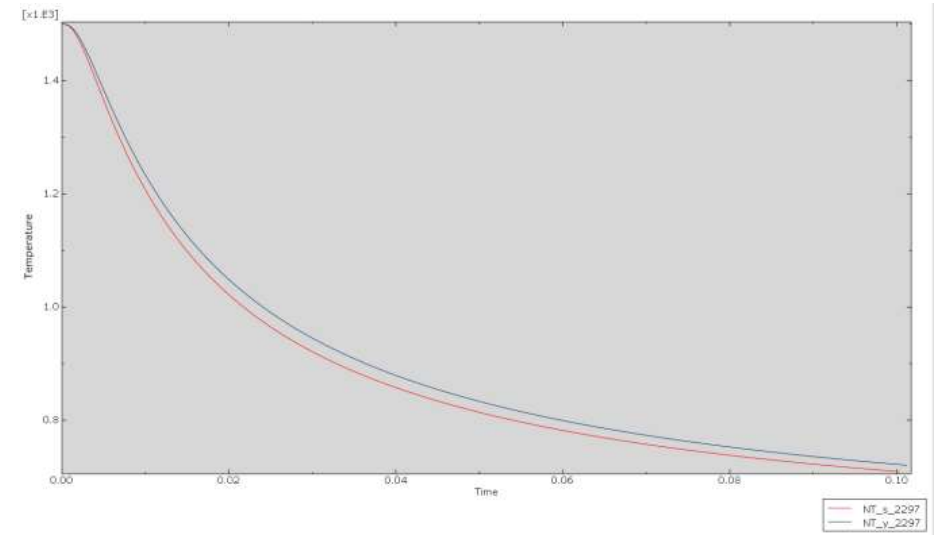

(a)

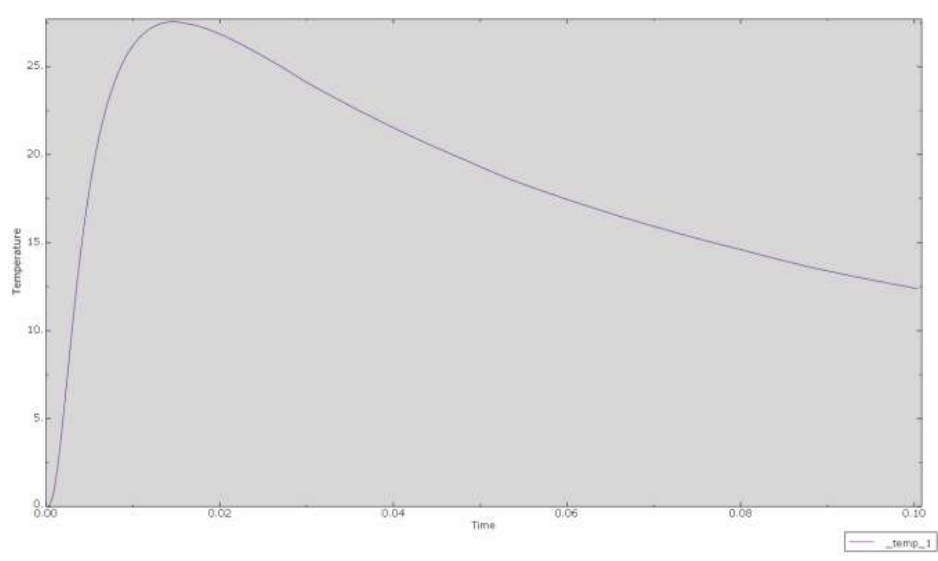

(b)

Fig. 9 (a) Temperature variation of the A-point original sample and graphene-added sample with time; (b) Temperature Difference Between A-point Sample and Graphene Sample with Time

Fig. 9 (a) shows that the temperature of the graphene sample is always higher than that of the original sample during heat transfer and cooling. Fig. 9 (b) shows that the temperature difference increases rapidly at the beginning and then decreases rapidly after reaching its maximum value.

\section{Conclusions}

The Rockwell hardness of WC-Co cemented carbide with graphene and carbon nanotubes is above 89 HRA, which exceeds the performance requirements of existing shield machine tools with the same composition. With the addition of graphene and carbon nanotubes, the bending strength of cemented carbide increases by approximately $50 \%$. After adding graphene and carbon nanotubes, the thermal conductivity of WC-Co cemented carbide increases, and when the graphene content reaches $0.12 \%$, the thermal conductivity increases to $9.86 \%$. The working temperature can thus be reduced to prevent the Co phase from melting in extreme cases. 


\section{Acknowledgement}

The financial support from the Opening Foundation of State Key Laboratory of Shield Machine and Boring Technology (2014-02) and The National Natural Science Foundation of China (51478146;51327802).

\section{References}

[1] Xuehong Liu, Aizhong Zou. Analysis and application of shield cemented carbide [A]. Superhard Material Engineering, 2016, 28(2).

[2] Ke Chu, Xiao-hu Wang, Fan Wang, Yu-biao Li, Da-jian Huang, Hong Liu,Wen-lin Ma, Feng-xia Liu, Hu Zhang.Largely enhanced thermal conductivity of graphene/copper composites with highly aligned graphene network.Carbon 127 (2018) 102-112.

[3] Jannica Heinrichs, Mikael Olsson, Karin Yvell, Staffan Jacobson. On the deformation mechanisms of cemented carbide in rock drilling. Fundamental studies involving sliding contact against a rock crystal tip. International Journal of Refractory Metals and Hard Materials 77 (2018) 141-151.

[4] M. Sribalaji, Aminul Islam, Biswajyoti Mukherjee, Mayank Kumar Pandey, Anup Kumar Keshri.Tailoring the thermal shock resistance of titanium carbide by reinforcement with tungsten carbide and carbon nanotubes.Ceramics International 44 (2018) 2552-2562.

[5] Shubin Ren, Jianhao Chen, Xinbo He, Xuanhui Qu .Effect of matrix-alloying-element chromium on the microstructure and properties of graphite flakes/copper composites fabricated by hotpressing sintering.Carbon 127 (2018) 412-423.

[6] Shaojiu Yan, Xiang Chen, Qihu Hong.Research Progress of Graphene Reinforced Aluminum Matrix Nanocomposites.Journal of Aeronautical Materials 2016, 36(3):57-70.

[7] Shourong Liu. Strength of WC-Co Cemented Carbide [J]. Cemented Carbide, 2002, 19(3):129-135.

[8] Haoliang Li, Sichang Dai, Jie Miao, Xian Wu, Navya Chandrasekharan, Hanxun Qiu,Junhe Yang.Enhanced thermal conductivity of graphene/polyimide hybrid film via a novel "molecular welding" strategy.Carbon 126 (2018) 319-327.

[9] Zicheng Ling, Cuixia Yan, Qingnan Shi, Zhongxue Feng. Effect of Ball Milling Time on Microstructure and Properties of Graphene/Copper Composites [J]. Rare Metal Materials And Engineering, 2017, 46(1):207-212.

[10] Guojun Zhou, Zhikai Ye, Weiwei Shi. Application of three-dimensional (3D) graphene and Its Composites [J]. Progress in Chemistry, 2014, 26(6):950-960.

[11] Peng Jin, Wen Peng. Study on thermal conductivity of cemented carbide [J]. Cemented 
Carbide, 2015, 32(5):300-305.

[12] Yulei Xing, Ke Yu, Yanhui Liu, Lingpin Zhang. Research on the progress of high thermal conduction mechanism and heat transfer enhancenment application of graphene [J]. Chemical Engineer, 2015, 29(5):54-60.

[13] Zeshao Chen, Jun Qian, Yihuo Ye. Theoretical Calculation of Equivalent Thermal Conductivity of Composites [J]. Journal of University of Science and Technology of China, 1992(4):416-424.

[14]Burak Cagri Ocak, Baris Yavas, Ipek Akin, Filiz Sahin, Gultekin Goller.Spark plasma sintered $\mathrm{Zr}$ C-Ti C-GNP composites: Solid solution formation and mechanical properties.Ceramics International 44 (2018) 2336-2344.

[15] Bohua Yu, Yunping Li, Qian Lei b, Yan Nie. Microstructuresand mechanical properties of WC-Co-xCr-Mo cement carbides. Journal of Alloys and Compounds 771 (2019) 636-642.

[16] Peng Qiu, Jiapan Zhou, Zhiwei Li. The preparation and electrochemical performance of graphene/carbon nanotube composite [J]. Carbon Techniques, 2017, 36(4):21-26.

[17] Ming Yan. Study on Creep-Thermal Fatigue Life Prediction [D]. Northeastern University, 2008.

[18] Williams, WS. The thermal conductivity of metallic ceramics. JOM-JOURNAL OF THE MINERALS METALS \& MATERIALS SOCIETY. 50 (1998) 62-66

[19] Nursel AltanÖzbek, AdemÇiçek, MahmutGülesin, OnurÖzbek. Investigation of the effects of cryogenic treatment applied at different holding times to cemented carbide inserts on tool wear. International Journal of Machine Tools and Manufacture 86 (2014) 34-43.

[20] Hongtao Wang, Tim Webb, Jonathan W. Bitler. Study of thermal expansion and thermal conductivity of cemented WC-Co composite. International Journal of Refractory Metals and Hard Materials 49 (2015) 170-177.

[21] Aziz Babapoor, Mehdi Shahedi Asl, Zohre Ahmadi, Abbas Sabahi Namini. Effects of spark plasma sintering temperature on densification, hardness and thermal conductivity of titanium carbide. Ceramics International 44 (2018) 14541-14546. 